\title{
Modelling an isolated dust grain in a plasma using matched asymptotic expansions
}

\author{
N. A R I N A M I N P A T H Y, J. E. A L L E N and J. R. OCK E N D O N \\ OCIAM, Mathematical Institute, Oxford OX1 3LB, UK \\ (nim.pathy@zoo.ox.ac.uk)
}

(Received 5 October 2006)

\begin{abstract}
The study of dusty plasmas is of significant practical use and scientific interest. A characteristic feature of dust grains in a plasma is that they are typically smaller than the electron Debye distance, a property which we exploit using the technique of matched asymptotic expansions. We first consider the case of a spherical dust particle in a stationary plasma, employing the Allen-Boyd-Reynolds theory, which assumes cold, collisionless ions. We derive analytical expressions for the electric potential, the ion number density and ion velocity. This requires only one computation that is not specific to a single set of dust-plasma parameters, and sheds new light on the shielding distance of a dust grain. The extension of this calculation to the case of uniform ion streaming past the dust grain, a scenario of interest in many dusty plasmas, is less straightforward. For streaming below a certain threshold we again establish asymptotic solutions but above the streaming threshold there appears to be a fundamental change in the behaviour of the system.
\end{abstract}

\section{Introduction}

Dust particles are commonplace in plasmas. When manufacturing semiconductors the presence of dust in plasmas can cause expensive damage to silicon substrates $[1,2]$ and yet also offers opportunities for the production of new, improved silicon films $[3,4]$. In astrophysics, dust plays an important role in the formation of stars and planets and in the constantly changing structure of planetary rings $[5,6]$. The extensive literature on the experimental and theoretical study of dusty plasmas has been summarized in a recent review [7].

When a dust grain is immersed in a plasma, it collects both ions and electrons. In the steady state it charges to a floating potential, such that the total ion current to its surface is balanced by the total electron current. Because electrons are more mobile than ions, this potential is typically negative. The floating potential depends fundamentally on the electron and ion dynamics and vice versa, and any solution to the dust-plasma problem needs to be determined self-consistently.

In the study of dusty plasmas the structure of the electric potential around a single grain is fundamental. In one experimental approach, Konopka et al. [8,9] studied head-on collisions between dust grains in the sheath of a radiofrequency (RF) discharge. By analysing the resulting grain trajectories, Konopka et al. calculated the grain charge and the screening length. They pointed out that theoretical work was required to determine 'the origin of the deduced screening distances'. 
However, their results apply to dust in the sheath of the discharge and in this paper we shall be concerned with dust in the plasma.

Other than the work of Konopka et al. there is a lack of experimental evidence for the structure of the electric potential around a dust grain. In part, this is the result of practical difficulties involved; typical laboratory probes are larger, by orders of magnitude, than the dust grains themselves. Hence most estimates of the potential distribution around a single grain in a plasma rely on theoretical models. These include kinetic particle-in-cell (PIC) simulations [10,11], as well as numerical studies of models such as the Allen-Boyd-Reynolds (ABR) theory (relevant to cold ions) [12] and the complete orbital motion theory (relevant to 'thermal' ions) [13].

In the present paper we first study the ABR model for small grains using the technique of matched asymptotic expansions [14]. In the following section we present the ABR model, non-dimensionalize it and then show that the problem is mathematically well-posed. Finally, in Sec. 4, we consider the effect of a uniform ion streaming relative to the grain.

\section{The ABR model}

Consider a spherical dust grain in a stationary plasma. In the steady state, the grain charges to a floating potential which is negative with respect to the plasma, so that ions are accelerated towards the grain and electrons repelled. We make the following assumptions about the plasma.

(1) The ions are singly charged, collisionless and 'cold', so that their net radial motion towards the grain dominates their random, thermal motion.

(2) The electrons are near thermal equilibrium, so that their density is accurately described by a Boltzmann distribution. This is likely to hold over most of space but fails near the grain surface, because of the collection of electrons there. We check the validity of this assumption a posteriori.

(3) We neglect ionization and recombination.

We note that, although we do not deal with collisions in this paper, a small number of collisions at great distances can, in some cases, result in ions moving essentially in the radial direction. This has been demonstrated in the case of Langmuir probe measurements [15] and similar behaviour is to be expected in the case of a dust particle. This is one of the reasons why the radial motion theory is of interest to experimental physicists.

We work in spherical co-ordinates, all variables depending only on the radial distance, $r$. We denote the grain radius by $R_{\text {dust }}$ and the electric potential by $V$. Taking the ions to have number density $N_{\mathrm{i}}$, mass $M_{\mathrm{i}}$ and speed $v_{\mathrm{i}}$, we have

$$
\begin{gathered}
\frac{1}{2} M_{\mathrm{i}} v_{\mathrm{i}}^{2}+e V=c, \\
\frac{d}{d r}\left(r^{2} N_{\mathrm{i}} v_{\mathrm{i}}\right)=0, \\
\frac{d^{2} V}{d r^{2}}+\frac{2}{r} \frac{d V}{d r}=-\frac{e}{\varepsilon_{0}}\left(N_{\mathrm{i}}-N_{\mathrm{e}}\right),
\end{gathered}
$$

where $e$ is the magnitude of the electron charge, $N_{\mathrm{e}}$ is their number density, $\varepsilon_{0}$ 
is the permittivity of free space and $c$ is a constant. These equations correspond, respectively, to the conservation of ion energy, conservation of ion flux and Poisson's equation. From assumption (2), $N_{\mathrm{e}}$ satisfies

$$
N_{\mathrm{e}}=N_{0} \exp \left(\frac{e V}{k_{\mathrm{B}} T_{\mathrm{e}}}\right),
$$

where $k_{\mathrm{B}}$ is Boltzmann's constant, $T_{\mathrm{e}}$ is the electron temperature and $N_{0}$ is the plasma density. On the grain surface the potential is not known a priori. Instead it obeys the floating condition, which states that the total ion flux to the surface is equal to the total electron flux, as derived in, for example, [16]. That is,

$$
-N_{\mathrm{i}} v_{\mathrm{i}}=N_{0} \sqrt{\frac{k_{\mathrm{B}} T_{\mathrm{e}}}{2 \pi m_{\mathrm{e}}}} \exp \left(\frac{e V}{k_{\mathrm{B}} T_{\mathrm{e}}}\right) \quad \text { on } r=R_{\text {dust }},
$$

where $m_{\mathrm{e}}$ is the electron mass. Owing to the radial symmetry of the model, (2.3) holds whether the grain is electrically conducting or an insulator. Finally, we impose plasma conditions far from the grain:

$$
V, v_{\mathrm{i}} \rightarrow 0, \quad N \rightarrow N_{0} \quad \text { as } r \rightarrow \infty
$$

These are the equations of the ABR theory [17], which was initially developed to model an electrostatic probe in a plasma, and was subsequently applied in the theory of dusty plasmas. The fact that the floating potential must be found as part of the solution raises questions about the well-posedness of the problem. From a physical point of view, the dust grain and the plasma interact to automatically set up a potential distribution in space, and a floating potential; hence, in a model capturing the essential interactions between the grain and the plasma, we would expect there to be enough information to specify the potential distribution uniquely.

We employ the following normalizations,

$$
\begin{aligned}
\hat{r} & =\frac{r}{\lambda_{\mathrm{De}}}, \\
\phi & =\frac{e V}{k T_{\mathrm{e}}}, \\
n & =\frac{N_{\mathrm{i}}}{N_{0}}, \\
n_{\mathrm{e}} & =\frac{N_{\mathrm{e}}}{N_{0}}, \\
q & =\frac{v_{\mathrm{i}}}{v_{\mathrm{B}}},
\end{aligned}
$$

where $v_{\mathrm{B}}=\sqrt{k_{\mathrm{B}} T_{\mathrm{e}} / M_{\mathrm{i}}}$, the Bohm speed, and we will subsequently drop the hat on $r$. Furthermore, we introduce a normalized dust radius

$$
\epsilon=\frac{R_{\text {dust }}}{\lambda_{\mathrm{De}}} .
$$


Note that, in a typical laboratory plasma, $\lambda_{\text {De }}$ can be of the order of millimetres and $R_{\text {dust }}$ of the order of tens of micrometres. Correspondingly, in this paper we will be concerned with the case $\epsilon \sim 10^{-2}$.

Using the above normalizations, we can eliminate $n$ and $q$ from the resulting system of equations to obtain

$$
\frac{d^{2} \phi}{d r^{2}}+\frac{2}{r} \frac{d \phi}{d r}=e^{\phi}-\frac{J}{r^{2} \sqrt{-2 \phi}},
$$

where $4 \pi J$, an unknown constant, is the total ion flux to the dust surface, normalized in terms of $\lambda_{\mathrm{De}}^{2} v_{\mathrm{B}} N_{0}$. Knowing $\phi$, we can determine $q$ and $n$ from

$$
\frac{1}{2} q^{2}+\phi=0, \quad r^{2} n q=J .
$$

Two boundary conditions are

$$
\phi \rightarrow 0 \text { as } r \rightarrow \infty
$$

and

$$
J=\epsilon^{2} \gamma e^{\phi(\epsilon)}
$$

where $\gamma$ is the 'gas parameter', given by

$$
\gamma=\sqrt{\frac{M_{\mathrm{i}}}{2 \pi m_{\mathrm{e}}}}
$$

and $n, q$ depend on $\phi$ through

$$
n=\frac{J}{r^{2} \sqrt{-2 \phi}}, \quad q=-\sqrt{-2 \phi} .
$$

From (2.4), a third boundary condition is

$$
n \rightarrow 1 \quad \text { as } r \rightarrow \infty \text {. }
$$

Note that, when we take $c=0$ in (2.1) without loss of generality, the condition $q \rightarrow 0$ as $r \rightarrow \infty$ is satisfied automatically by (2.9). There are thus two specified parameters in this problem, $\epsilon$ and $\gamma$, and $\epsilon \sim 0.01$, whereas $\gamma$ is at least 17 for hydrogen and can be as large as 108 in the typical case of argon.

Returning now to the question of uniqueness, because $J$ and $\phi(\epsilon)$ are unknown a priori, it seems that there is insufficient information to determine $\phi$ uniquely. However, the condition (2.13) is more powerful than it might initially seem; it allows us ultimately to associate a unique value of $J$ with any choice of $\epsilon$ and $\gamma$, as we now show.

First, we consider the related problem where $J$ is known, but we drop the floating condition (2.10). We then have the following theorem, a proof of which is given in Appendix A.

TheOREm 2.1 (Uniqueness ReSUlt). If a solution exists to the problem

$$
\frac{d^{2} \phi}{d r^{2}}+\frac{2}{r} \frac{d \phi}{d r}=e^{\phi}-\frac{J}{r^{2} \sqrt{-2 \phi}}, \quad \phi<0, \epsilon<r<\infty,
$$

such that

$$
\frac{J}{r^{2} \sqrt{-2 \phi}} \rightarrow 1 \text { as } r \rightarrow \infty,
$$

where $J$ is a given, positive constant, then that solution is unique, for any $\epsilon>0$. 
To address the fact that $J$ is unknown, we now incorporate the floating condition (2.10) using the sequence

$$
J \longmapsto \phi(r) \stackrel{\epsilon}{\longmapsto} \phi(\epsilon) \stackrel{\epsilon, \gamma}{\longmapsto} J
$$

where ' $A \stackrel{C}{\longmapsto} B$ ' states that $B$ follows uniquely from $A$, given $C$. The first arrow in (2.16) follows from the uniqueness theorem, and the last arrow follows from the floating condition (2.10). Hence we expect ultimately to find an equation for $J$, and hence $\phi(r)$, for any given $\epsilon, \gamma$.

It is convenient to define the shorthand notation

$$
\hat{\epsilon}=\epsilon \gamma^{1 / 2}
$$

and

$$
k=e^{\phi(\epsilon)},
$$

so that $\hat{\epsilon}$ is proportional to the dust size, and $k$ is a measure of the unknown surface potential. Hence, using the floating condition, we have

$$
J=\hat{\epsilon}^{2} k \text {. }
$$

Note that $\hat{\epsilon}$ is a known quantity, whereas $k$ is unknown. Typically, $\epsilon=0.01, \gamma \lesssim 10^{2}$, so that we assume

$$
\hat{\epsilon} \ll 1 \text {. }
$$

Because the surface potential is negative we have from $(2.18)$ that $0<k<1$. Moreover, it has been shown [12] that the floating potential goes to zero as $\epsilon \rightarrow 0$, and so we assume here that

$$
k \sim O(1)
$$

and we will verify this a posteriori.

In terms of $\hat{\epsilon}, k,(2.16)$ can be written as an iteration to determine $k$ :

$$
k \stackrel{\hat{\epsilon}}{\longmapsto} \phi(r) \stackrel{\hat{\epsilon}, \gamma}{\longmapsto} \phi\left(\hat{\epsilon} \gamma^{-1 / 2}\right) \stackrel{\hat{\epsilon}, \gamma}{\longmapsto} k,
$$

where

$$
\frac{d^{2} \phi}{d r^{2}}+\frac{2}{r} \frac{d \phi}{d r}=e^{\phi}-\frac{\hat{\epsilon}^{2} k}{r^{2} \sqrt{-2 \phi}}, \quad \hat{\epsilon} \ll 1, \hat{\epsilon} \gamma^{-1 / 2}<r<\infty,
$$

for $\phi, k$ with

$$
\phi \sim-\hat{\epsilon}^{4} k^{2} / 2 r^{4} \quad \text { as } r \rightarrow \infty
$$

and

$$
k=\exp \left(\phi\left(\hat{\epsilon} \gamma^{-1 / 2}\right)\right)
$$

Although our basic assumption is that $\hat{\epsilon}$ is small, we must remember that $\gamma$ may be as large as $O\left(10^{2}\right)$, so that we are confronted with a two-parameter asymptotic problem. However, for ease of presentation we will begin by taking $\gamma=O(1)$.

\section{Matched asymptotic expansions}

In order to find $\phi(r)$ asymptotically for fixed $\gamma=O(1)$ as $\hat{\epsilon} \rightarrow 0$ for any $k=O(1)$, we find it convenient to begin with the far-field expansions, valid as $r \rightarrow \infty$. Working inwards to the dust surface, we ultimately use (2.25) to determine $k$. 
On physical grounds, referring to Poisson's equation $\nabla^{2} \phi=e^{\phi}-n$, we expect that far from the grain we would have a quasi-neutral plasma region where $\nabla^{2} \phi \ll e^{\phi}, n$. We shall refer to this as the 'outer region'. Closer to the grain, we expect to find a space-charge region where $\nabla^{2} \phi \sim O\left(e^{\phi}, n\right)$, in which there is a balance of terms in Poisson's equation. We shall refer to this as the 'intermediate region'. We use matching (as described by Van Dyke [14]) to ensure that the asymptotic expansions found in each of these regions are mutually consistent, and this will also reveal the existence of an inner expansion, for $r \sim O(\hat{\epsilon})$, where the space charge is merely a small perturbation in the determination of the electric potential.

\subsection{Outer region}

From (2.24) we have that

$$
\phi \sim-\frac{\hat{\epsilon}^{4} k^{2}}{2 r^{4}}+\bar{\phi} \quad \text { as } r \rightarrow \infty
$$

where

$$
\hat{\epsilon}^{4} k^{2} \nabla^{2} \bar{\phi}+r^{4} \bar{\phi} \sim \frac{\hat{\epsilon}^{8} k^{4}}{2 r^{4}}\left(\frac{12}{r^{2}}-1\right) \quad \text { as } r \rightarrow \infty .
$$

This gives that

$$
\bar{\phi} \sim \frac{\hat{\epsilon}^{8} k^{4}}{2 r^{8}}\left(\frac{12}{r^{2}}-1\right)+\cdots \quad \text { as } r \rightarrow \infty
$$

i.e.

$$
\phi \sim-\frac{\hat{\epsilon}^{4} k^{2}}{2 r^{4}}+\frac{\hat{\epsilon}^{8} k^{4}}{2 r^{8}}\left(\frac{12}{r^{2}}-1\right)+\cdots \quad \text { as } r \rightarrow \infty .
$$

We also have

$$
n \sim 1+\frac{\hat{\epsilon}^{4} k^{2}}{2 r^{4}}\left(\frac{12}{r^{2}}-1\right)+\cdots, \quad q \sim-\frac{\hat{\epsilon}^{2} k}{r^{2}}+\frac{\hat{\epsilon}^{6} k^{3}}{2 r^{6}}\left(\frac{12}{r^{2}}-1\right)+\cdots .
$$

The expansion (3.4) fails when the first and second terms become comparable, which occurs at a distance

$$
r \sim O\left(\hat{\epsilon}^{2 / 3}\right) .
$$

We also note that, on this scale, we have

$$
\nabla^{2} \phi \sim O\left(\frac{\hat{\epsilon}^{4} / \hat{\epsilon}^{8 / 3}}{\hat{\epsilon}^{4 / 3}}\right) \sim O(1), \quad n, e^{\phi} \sim O(1),
$$

indicating that space charge has become significant. Hence we enter the intermediate region.

\subsection{Intermediate region $r \sim O\left(\hat{\epsilon}^{2 / 3}\right)$}

We use capital letters to denote terms in the intermediate expansions. Referring to the outer expansions, we find that $\phi \sim O\left(\hat{\epsilon}^{4 / 3}\right)$ when $r \sim O\left(\hat{\epsilon}^{2 / 3}\right)$. Thus we rescale by writing

$$
r=\hat{\epsilon}^{2 / 3} R, \quad \phi \sim \hat{\epsilon}^{4 / 3} \bar{\phi}_{0}(R)+\cdots .
$$

Recall, as noted earlier, that the dust surface is in the region $r \sim O(\hat{\epsilon})$, i.e. on a smaller length scale than the intermediate region. Thus, to find the behaviour of $\phi$ in the vicinity of the dust grain we will be interested in the small- $R$ behaviour of the intermediate expansions defined above. 
Now, substituting (3.8) into (2.23) and letting $\hat{\epsilon} \rightarrow 0$ yields the lowest-order equation

$$
\frac{d^{2} \bar{\phi}_{0}}{d R^{2}}+\frac{2}{R} \frac{d \bar{\phi}_{0}}{d R}=1-\frac{k}{R^{2} \sqrt{-2 \bar{\phi}_{0}}}, \quad 0<R<\infty,
$$

with the far-field matching condition

$$
\bar{\phi}_{0} \sim-\frac{k^{2}}{2 R^{4}} \quad \text { as } R \rightarrow \infty,
$$

which ensures the consistency, to lowest order, of the intermediate and outer expansions.

Equation (3.9) is almost the full problem, apart from the electron density term being replaced by 1 . Hence, with a proof similar to that of the uniqueness result we can show that, for a given $k,(3.9)$ together with (3.10) has a unique solution for $\bar{\phi}_{0}$. The problem must be solved numerically. To do this we can fortunately scale $k$ out of the problem by writing

$$
R=k^{1 / 3} \tilde{r}, \quad \bar{\phi}_{0}(R)=k^{2 / 3} \tilde{\phi}_{0}(\tilde{r}) .
$$

The problem now reads

$$
\frac{d^{2} \tilde{\phi}_{0}}{d \tilde{r}^{2}}+\frac{2}{\tilde{r}} \frac{d \tilde{\phi}_{0}}{d \tilde{r}}=1-\frac{1}{\tilde{r}^{2} \sqrt{-2 \tilde{\phi}_{0}}}
$$

with

$$
\tilde{\phi}_{0} \sim-\frac{1}{2 \tilde{r}^{4}} \quad \text { as } \tilde{r} \rightarrow \infty .
$$

To work towards the dust surface we now find the behaviour of $\tilde{\phi}_{0}$ in the small-r limit. Since

$$
\frac{d^{2}\left(\tilde{r} \tilde{\phi}_{0}\right)}{d \tilde{r}^{2}}=\tilde{r}-\frac{1}{\sqrt{-2 \tilde{r}\left(\tilde{r} \tilde{\phi}_{0}\right)}}
$$

with

we find that

$$
\tilde{r} \tilde{\phi}_{0} \sim-\frac{1}{2 \tilde{r}^{3}} \quad \text { as } \tilde{r} \rightarrow \infty
$$

$$
\tilde{\phi}_{0} \sim \frac{c_{0}}{\tilde{r}}+c_{1}-\frac{4}{3}\left(-2 c_{0}\right)^{-1 / 2} \tilde{r}^{1 / 2}+\cdots \quad \text { as } \tilde{r} \rightarrow 0,
$$

for constants $c_{0}, c_{1}$, both fixed by (3.15). The coefficients of all successive terms are determined by the constants $c_{0}, c_{1}$. To find these constants, we carry out a numerical integration of (3.14). When written in terms of $\psi=\tilde{r} \tilde{\phi}_{0}$ we shoot from some large $\tilde{r}$, using there the initial conditions on $\psi, d \psi / d \tilde{r}$ provided by (3.15), and then estimate

$$
c_{0}=\lim _{\tilde{r} \rightarrow 0} \psi, \quad c_{1}=\lim _{\tilde{r} \rightarrow 0} \psi^{\prime} .
$$

Note that $d^{2} \psi / d \tilde{r}^{2}$ is unbounded in this limit, but we can even work down to $\tilde{r}=10^{-12}$ to obtain reasonable estimates of $c_{0}$ and $c_{1}$, as shown in Fig. 1 . Thus we find

$$
c_{0} \approx-0.98, \quad c_{1} \approx 1.71
$$




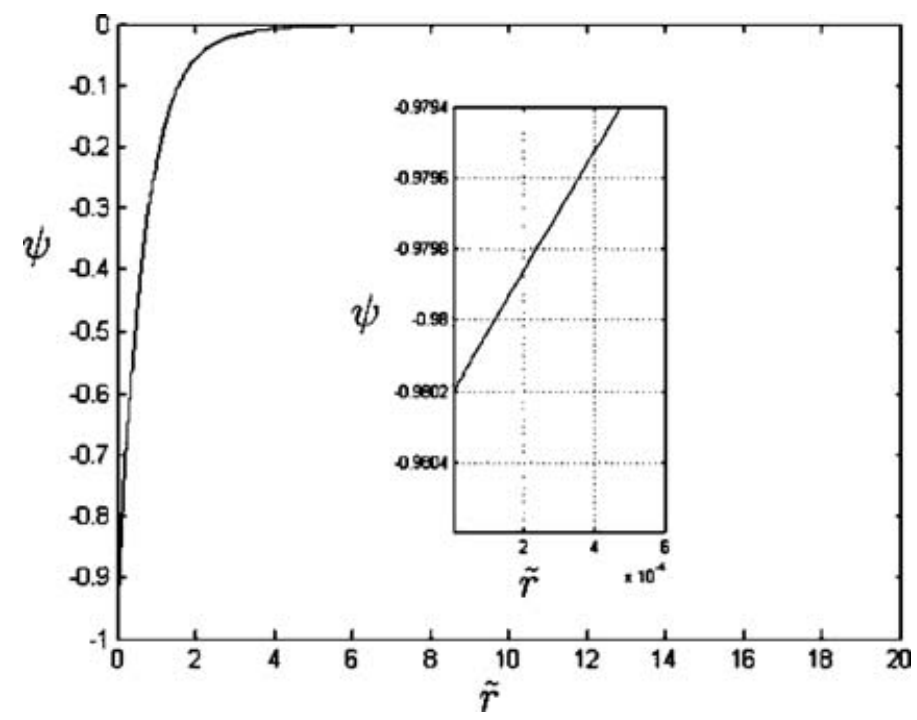

Figure 1. Numerical plot of $\psi(\tilde{r})$. The inset shows a magnification near $\tilde{r}=0$, with both axes on the same scale. The integration parameters are $\tilde{r}_{\text {init }}=40, \tilde{r}_{\text {end }}=10^{-12}$.

Note that, because both $\hat{\epsilon}$ and $k$ have been scaled out of the problem on this length scale, the constants $c_{0}, c_{1}$ are universal.

Thus, in terms of the original intermediate variables defined in (3.8) we have

$$
\bar{\phi}_{0} \sim \frac{c_{0} k}{R}+\cdots \quad \text { as } R \rightarrow 0,
$$

and $n, q$ are given by

$$
n \sim \frac{k}{R^{2} \sqrt{-2 \bar{\phi}_{0}}}+\cdots, \quad q \sim-\hat{\epsilon}^{2 / 3} \sqrt{-2 \bar{\phi}_{0}}+\cdots .
$$

From (2.1b), we note that this implies that the divergence of the ion flux $n q$ satisfies

$$
\frac{d}{d \tilde{r}}(\tilde{n} \tilde{q})+\frac{2 \tilde{n} \tilde{q}}{\tilde{r}}=-4 \pi \delta(\tilde{\mathbf{r}}),
$$

to lowest order in the intermediate region, where

$$
n=k \tilde{n}, \quad q=\hat{\epsilon}^{2 / 3} k^{1 / 3} \tilde{q} .
$$

This region thus perceives the dust particle as an ion sink of strength $4 \pi$.

In principle, we should now make a final rescaling in which $r \sim O(\hat{\epsilon})$ before using the information in (2.25). However, were we to scale $\phi$ and $r$ with $\hat{\epsilon}$ we would simply find that $\phi$ satisfied Laplace's equation to lowest order and the relevant solution would simply be the first term in (3.19) and (3.20). Hence, in outer variables, the solution near the grain is

$$
\phi \sim \frac{\hat{\epsilon}^{2} c_{0} k}{r}+\cdots, \quad n \sim \frac{\hat{\epsilon} \sqrt{k}\left(-2 c_{0}\right)^{-1 / 2}}{r^{3 / 2}}+\cdots, \quad q \sim \frac{-\hat{\epsilon} \sqrt{k}\left(-2 c_{0}\right)^{1 / 2}}{r^{1 / 2}}+\cdots,
$$

and we now come to the vital closure condition on $k$. 
Setting $r=\hat{\epsilon} \gamma^{-1 / 2}$ in (3.23), we find that the surface potential is

$$
\phi(\epsilon) \sim \epsilon \gamma c_{0} k,
$$

and hence $k$ is given, to lowest order, by

$$
k \sim \exp \left(\epsilon \gamma c_{0} k\right) .
$$

Since $c_{0}<0,(3.25)$ has a unique solution $k$. Moreover we see that, since $\gamma$ has been assumed to be $O(1), k \sim 1$ to lowest order. However, since our asymptotic procedure is valid as long as both $\hat{\epsilon}=\epsilon \gamma^{1 / 2} \ll 1$ and $k=O(1)$, we see that (3.25) gives a correct, unique value of $k$ to lowest order even when $\gamma \gg 1$, as long as $\epsilon \gamma \lesssim O(1)$. This is true in most physically realistic cases, as, for example, in laboratory experiments using argon where $\epsilon=0.01, \gamma=108$. In this case we find $k \approx 0.56$. For comparison, a full numerical calculation was also conducted on (2.7)-(2.10) using a shooting method [18], giving

$$
k=0.57 \text {. }
$$

Some potential distributions are presented in Fig. 2, which shows $\phi(r)$ for two different particle sizes, $\epsilon=0.01$ and $\epsilon=0.0001$. In these graphs the asymptotic and full numerical solutions are superimposed for comparison.

We now briefly discuss some physical implications of this calculation.

\subsection{Electron density}

The electron Boltzmann relation (2.2) arises from integrating the MaxwellBoltzmann density distribution function over the entire velocity space [19]. We now consider the effect of an absorbing dust grain on the validity of this procedure.

Being negatively charged, the dust grain repels electrons but at any point outside the dust grain a certain proportion of electrons moving towards the dust grain will have sufficient energy to reach the dust surface and be collected there. This will introduce a deficit in the number of electrons moving radially away from the grain and hence introduce an error in the Boltzmann distribution there. On length scales larger than the grain, i.e. $r \gg \epsilon$, the grain appears as a localized sink, and the proportion of electrons reaching the grain is small. Hence the error in the Boltzmann distribution is also small, whenever $r \gg \epsilon$.

On the other hand, sufficiently close to the grain the Boltzmann distribution is invalid; adjacent to the surface, for example, there are electrons moving towards the surface but none are travelling away, and so the right-hand side of $(2.2)$ is an overestimate by a factor of 2 . However, this does not invalidate our results because, as discussed after (3.20), $\phi$ satisfies Laplace's equation in the inner region, to lowest order. Physically, this is because the potential distribution near the sphere surface is predominantly the result of charge on the surface itself, rather than the surrounding free electrons and ions. Thus the lowest-order expansion in $\phi$ would be unaffected by correcting overestimates in the electron density, and hence our solutions remain valid.

\subsection{Shielding}

A new shielding distance has arisen naturally from the calculation. The scale $r \sim \epsilon^{2 / 3} \gamma^{1 / 3}$ derived in (3.6) corresponds physically to the size of the space-charge region, and therefore represents the shielding distance. In 'unnormalized' variables 


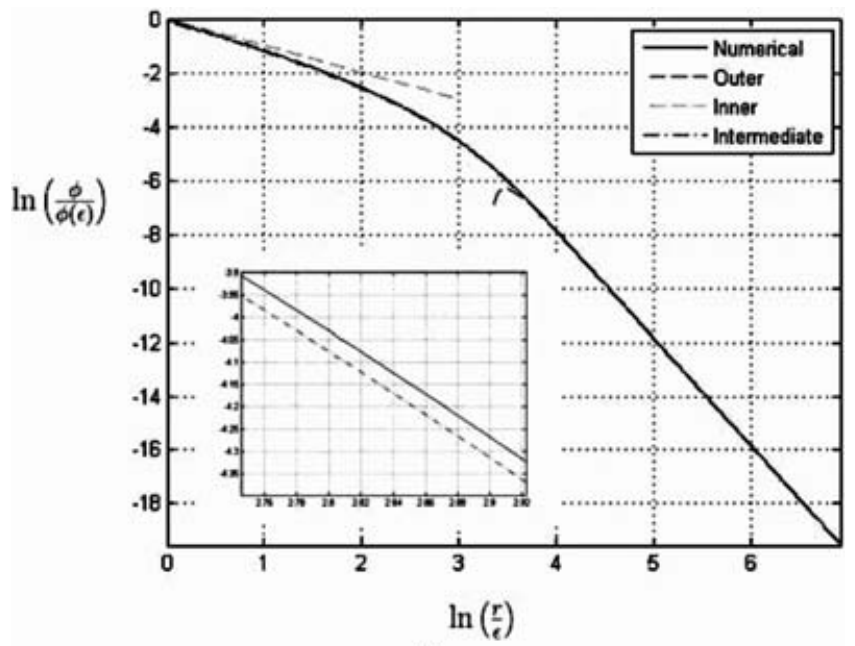

(a)

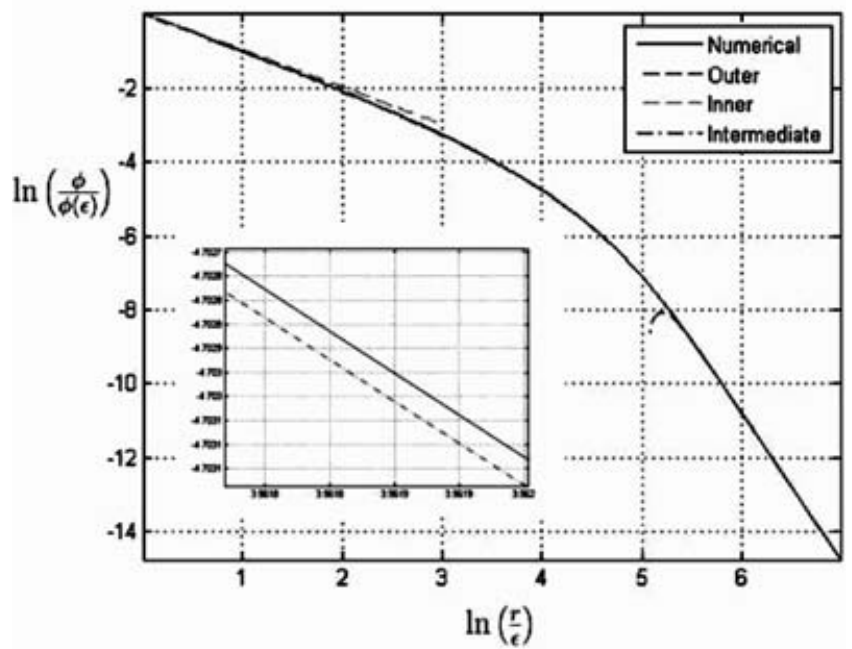

(b)

Figure 2. Comparison of inner, intermediate and outer MAE solutions (dashed lines), with 'full' numerical solution (solid line), for different particle sizes: (a) $\epsilon=0.01, \gamma=108$; (b) $\epsilon=0.001, \gamma=108$. On the scale of both graphs the numerical 'intermediate' and 'full' solutions are indistinguishable. The inset shows a close-up of these two plots in the intermediate region. The surface potentials are (a) $\phi(\epsilon)=-0.56$ and (b) $\phi(\epsilon)=-0.01$.

this distance is

$$
R_{\mathrm{s}} \sim R_{\text {dust }}^{2 / 3} \gamma^{1 / 3} \lambda_{\text {De }}^{1 / 3} .
$$

We recall the two shielding distances commonly quoted in the literature, namely the 'electron' and the so-called 'linearized' Debye lengths, respectively,

$$
\lambda_{\mathrm{De}}=\sqrt{\frac{\varepsilon_{0} k_{\mathrm{B}} T_{\mathrm{e}}}{e^{2} N_{0}}}, \quad \lambda_{\mathrm{Dl}}=\left(\frac{e^{2} N_{0}}{\varepsilon_{0} k_{\mathrm{B}} T_{\mathrm{e}}}+\frac{e^{2} N_{0}}{\varepsilon_{0} k_{\mathrm{B}} T_{\mathrm{i}}}\right)^{-1 / 2} .
$$


Consider first the electron Debye distance. Under the normalization in (2.5a) it is unity, which is clearly larger than our new estimate: in the case of argon, for example, when $\epsilon=0.01$ we have $R_{\mathrm{s}} / \lambda_{\text {De }}=\epsilon^{2 / 3} \gamma^{1 / 3} \sim 0.2$. Although $\lambda_{\text {De }}$ can be a characteristic length scale in its own right, it does not necessarily correspond to the shielding distance of a dust grain. On the other hand, the 'linearized' Debye distance arises from the Debye-Huckel model. This assumes that the ions are in thermal equilibrium and, in the case of finite $T_{\mathrm{i}}$, when the ions are collisionless and $R_{\text {dust }} / \lambda_{\mathrm{Dl}} \ll 1$, then the potential distribution predicted by this model is an excellent approximation, except at large distances [13]. However, for our cold-ion scenario, the theory is inapplicable. In particular, allowing $T_{\mathrm{i}} \rightarrow 0$ in $\lambda_{\mathrm{Dl}}$ in (3.28) results in the Debye length also going to zero, which is unphysical for the case of a dust grain in a plasma with cold ions.

Apart from the possible numerical discrepancies between (3.27) and (3.28), we emphasize that our new shielding distance for a dust grain in a cold plasma depends quite sensitively on the radius of the grain.

We now extend the radial-motion model to describe the effect of an ion stream past the dust grain, a common scenario in dusty plasmas.

\section{Uniform ion streaming}

We write $\nu$ for the steady ion streaming speed, normalized in terms of the Bohm speed $v_{\mathrm{B}}$. In Cartesian co-ordinates we assume that the streaming is parallel to the $x$-axis, with the dust grain centred at the origin. We no longer have spherical symmetry, but rather axial symmetry about the $x$-axis. Denoting the dimensionless ion velocity by $\mathbf{q}$ and using the same non-dimensional variables as in (2.5) we then have

$$
\begin{gathered}
\nabla \cdot(n \mathbf{q})=0, \\
(\mathbf{q} \cdot \nabla) \mathbf{q}=-\nabla \phi, \\
\nabla^{2} \phi=e^{\phi}-n,
\end{gathered}
$$

corresponding, respectively, to conservation of ion flux, the equation of motion of the ions and Poisson's equation. The plasma streaming condition is

$$
\mathbf{q} \rightarrow \nu \hat{\mathbf{x}}, \quad \phi \rightarrow 0, \quad n \rightarrow 1 \quad \text { as } r \rightarrow \infty,
$$

where $\hat{\mathbf{x}}$ is the unit vector in the $x$-direction, and the floating condition on the grain surface $r=\epsilon$ is given by the following equations:

- conducting grain, $\phi=\phi_{\mathrm{d}}$ (constant), such that

$$
\left.\frac{1}{2} \int_{0}^{\pi}(-n \mathbf{q} \cdot \mathbf{n})\right|_{r=\epsilon} \sin \theta d \theta=\gamma e^{\phi_{d}}
$$

- insulated grain,

$$
-n \mathbf{q} \cdot \mathbf{n}=\gamma e^{\phi}
$$

where $\mathbf{n}$ is the unit outward normal to the grain. For ease of presentation we will again begin by assuming $\gamma$ is of $O(1)$.

Because the ions are initially irrotational, and move under a conservative force, by Kelvin's theorem the ion motion must remain irrotational throughout. Hence 
we can write $\mathbf{q}$ as the gradient of a velocity potential $\Phi$,

$$
\mathbf{q}=\nabla \Phi .
$$

Thus the far-field condition (4.2) on $\mathbf{q}$ corresponds to the requirement that $\Phi \rightarrow \nu x$ as $r \rightarrow \infty$. Also, we can replace (4.1b) by the energy equation

$$
\frac{1}{2}|\mathbf{q}|^{2}+\phi=\frac{1}{2} \nu^{2}
$$

\subsection{Ion flux in the outer region}

The dust grain transmits information about its presence even at large distances by establishing an ion flux to its surface. Integrating (4.1a) and using the divergence theorem and either (4.3) or (4.4) shows that the lowest-order correction to the uniform ion stream is a sink of strength $4 \pi \epsilon^{2} \gamma K=4 \pi \hat{\epsilon}^{2} K$, where $\hat{\epsilon}$ is as defined in $(2.17)$ and

$$
K=\left.\frac{1}{2} \int_{0}^{\pi} e^{\phi}\right|_{r=\epsilon} \sin \theta d \theta
$$

We now assume that, for small $\nu$,

$$
\Phi \sim \nu x+\frac{\hat{\epsilon}^{2} K}{r}+\cdots \quad \text { as } r \rightarrow \infty .
$$

The remaining governing equations yield the following expansions for $\phi, n$ valid for any $\hat{\epsilon}, \nu \ll 1$ :

$$
\begin{gathered}
\Phi \sim \nu x+\frac{\hat{\epsilon}^{2} K}{r}+\frac{\hat{\epsilon}^{2} \nu^{2} K}{4 r}\left(\frac{2 x^{2}}{r^{2}}-1\right)+\cdots, \\
\phi \sim \frac{\hat{\epsilon}^{2} \nu K x}{r^{3}}+\frac{\hat{\epsilon}^{2} \nu^{3} K x}{4 r^{3}}\left(\frac{6 x^{2}}{r^{2}}-5\right)-\frac{\hat{\epsilon}^{4} K^{2}}{2 r^{4}}+\cdots, \\
n \sim 1+\frac{\hat{\epsilon}^{2} \nu K x}{r^{3}}+\frac{\hat{\epsilon}^{2} \nu^{3} K x}{4 r^{3}}\left(\frac{6 x^{2}}{r^{2}}-5\right)+\cdots \quad \text { as } r \rightarrow \infty .
\end{gathered}
$$

Note that the effect of any non-zero streaming is to introduce a dipole into the electric field, far from the grain. However, the asymptotic behaviour nearer the dust grain, and hence the value of $K$, is different for different parameter regimes in $\nu$.

\subsection{Case 1: weak streaming, $0<\nu \ll \hat{\epsilon}^{2 / 3}$}

Equations (4.9)-(4.11) describe the lowest-order solution when we are sufficiently far from the dust particle for it to represent a small perturbation to the uniform ion stream, i.e. when $r \gg \hat{\epsilon} \nu^{-1 / 2}$. Even when $r \lesssim \hat{\epsilon} \nu^{-1 / 2}$ these expansions can still be carried out, with the ion stream acting as a small perturbation to the ABR solution in the form

$$
\Phi \sim \frac{\hat{\epsilon}^{2} K}{r}+\nu x+\cdots, \quad \phi \sim-\frac{\hat{\epsilon}^{4} K^{2}}{2 r^{4}}+\frac{\hat{\epsilon}^{2} \nu K x}{r^{3}}+\cdots, \quad n \sim 1+\frac{6 \hat{\epsilon}^{4} K^{2}}{r^{6}}+\cdots .
$$

However, when we further reduce $r$, we see that the outer expansions break down at a distance $r \sim O\left(\hat{\epsilon}^{2 / 3}\right)$ from the grain, the size of the space-charge region. In this region the ions have a kinetic energy considerably exceeding their original kinetic 


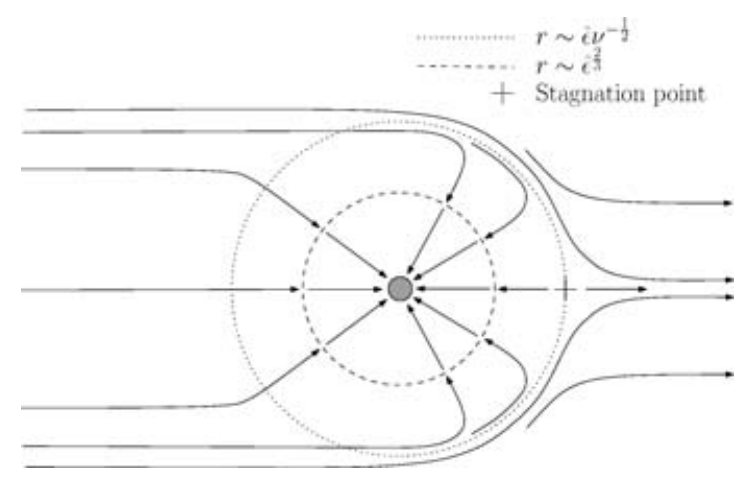

Figure 3. Spatial structure and streamlines for the 'weak stream' case.

energy, and we employ the rescalings

$$
\begin{aligned}
& r=\left(\hat{\epsilon}^{2 / 3} K^{1 / 3}\right) \tilde{r}, \quad \phi \sim\left(\hat{\epsilon}^{4 / 3} K^{2 / 3}\right) \tilde{\phi}_{0}+\cdots, \\
& n \sim \tilde{n}_{0}+\cdots, \quad \Phi \sim\left(\hat{\epsilon}^{4 / 3} K^{2 / 3}\right) \tilde{\Phi}_{0}+\cdots,
\end{aligned}
$$

where the terms in $K$ are again introduced to ensure that the lowest-order problem in this region is independent of $K$. Thus, for $\tilde{r}>0$, the lowest-order problem reads

$$
\begin{gathered}
\nabla \cdot\left(\tilde{n}_{0} \nabla \tilde{\Phi}_{0}\right)=0, \\
\frac{1}{2}\left|\nabla \tilde{\Phi}_{0}\right|^{2}+\tilde{\phi}_{0}=0, \\
\nabla^{2} \tilde{\phi}_{0}=1-\tilde{n}_{0},
\end{gathered}
$$

with matching conditions

$$
\begin{gathered}
\tilde{\Phi}_{0} \sim \frac{1}{\tilde{r}}, \\
\tilde{\phi}_{0} \sim-\frac{1}{2 \tilde{r}^{4}}, \\
\tilde{n_{0}} \rightarrow 1 \quad \text { as } \tilde{r} \rightarrow \infty .
\end{gathered}
$$

In particular, as in (3.21) we expect (4.14a) to imply that in the intermediate region

$$
\nabla \cdot\left(\tilde{n}_{0} \nabla \tilde{\Phi}_{0}\right)=-4 \pi \delta(\tilde{\mathbf{r}}) .
$$

The matching conditions (4.15) are the same as for the intermediate region in the radial-motion model. As indicated by the absence of $\nu$ in (4.14) and (4.15), the ion stream is too small to have any effect on the lowest-order terms in this region. Hence we expect that the lowest-order intermediate solutions are in fact spherically symmetric, giving the simple result that the intermediate and inner solutions are the same as in the radial-motion case.

Figure 3 shows schematically the ion streamlines in this case; whereas flowrelated terms dominate in the expansions at large distances, there is a 'switchover distance' $r \sim \hat{\epsilon} \nu^{-1 / 2}$ at which they are replaced at lowest order by spherically symmetric terms. As long as this distance is greater than the shielding distance, 


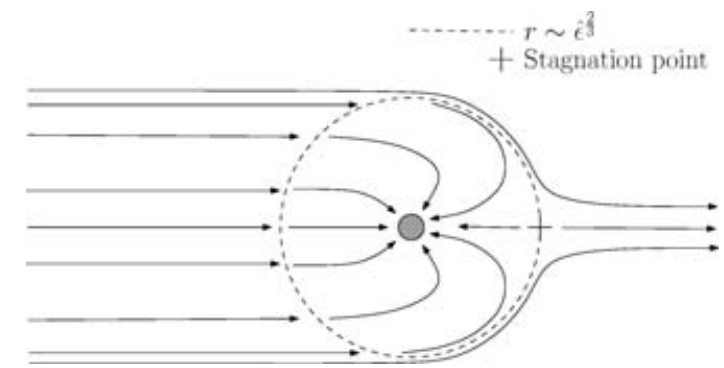

Figure 4. Spatial structure and streamlines for the 'threshold stream' case.

we revert to spherically symmetric lowest-order solutions from the intermediate region inwards.

Hence, to lowest order in $\nu, \phi$ is again constant on the grain and $K$ is equal to the value of $k$ calculated in Sec. 3. Moreover as discussed at the end of that section, this result remains true when $\gamma$ is as large as $\epsilon^{-1 / 2}$. However, by increasing $\nu$ we narrow the region between the switchover distance and the shielding distance, eventually reaching the case of threshold streaming.

\subsection{Case 2: threshold streaming, $\nu \sim O\left(\hat{\epsilon}^{2 / 3}\right)$}

The shielding length is once again $r \sim O\left(\hat{\epsilon}^{2 / 3}\right)$. We define

$$
\nu=\left(\hat{\epsilon}^{2 / 3} K^{1 / 3}\right) \tilde{\nu},
$$

and otherwise employ the same rescalings as in (4.13) and the idea in (4.16). We thus find, for the lowest-order 'canonical' problem,

$$
\begin{gathered}
\nabla \cdot\left(\tilde{n}_{0} \nabla \tilde{\Phi}_{0}\right)=-4 \pi \delta(\tilde{\mathbf{r}}), \\
\frac{1}{2}\left|\nabla \tilde{\Phi}_{0}\right|^{2}+\tilde{\phi}_{0}=\frac{1}{2} \tilde{\nu}^{2}, \\
\nabla^{2} \tilde{\phi}_{0}=1-\tilde{n}_{0},
\end{gathered}
$$

where the tilde denotes rescaled variables. The matching conditions are

$$
\begin{gathered}
\tilde{\Phi}_{0} \rightarrow \tilde{\nu} \tilde{x}, \\
\tilde{\phi}_{0} \rightarrow \frac{\tilde{\nu} \tilde{x}}{\tilde{r}^{3}}, \\
\tilde{n}_{0} \rightarrow 1 \quad \text { as } \tilde{r} \rightarrow \infty .
\end{gathered}
$$

Thus the lowest-order spherical symmetry inside the space-charge region has been broken by the ion flow. Although this problem cannot be solved analytically, when $\nu$ is sufficiently small we conjecture that the streamlines are as illustrated schematically in Fig. 4.

We can also make some conjecture about the regime $\nu \gg \hat{\epsilon}^{2 / 3}$, corresponding to $\tilde{\nu}$ becoming large. In particular, there is reason to doubt that the ion trajectory pattern in Fig. 4 is maintained for such values of $\tilde{\nu}$, by virtue of the following argument. 
It is possible to rescale $(4.18)$ by writing

$$
\begin{gathered}
\tilde{\Phi}_{0}=\tilde{\nu}^{1 / 2} \hat{\Phi}_{0}, \\
\tilde{\phi}_{0}=\tilde{\nu}^{2} \hat{\phi}_{0}, \\
\tilde{r}=\tilde{\nu}^{-1 / 2} \hat{r},
\end{gathered}
$$

to obtain

$$
\begin{gathered}
\nabla \cdot\left(n_{0} \nabla \hat{\Phi}_{0}\right)=-4 \pi \delta(\hat{\mathbf{r}}), \\
\frac{1}{2}\left|\nabla \hat{\Phi}_{0}\right|^{2}+\hat{\phi}_{0}=\frac{1}{2}, \\
\tilde{\nu}^{3} \nabla^{2} \hat{\phi}_{0}=1-n_{0} .
\end{gathered}
$$

The matching conditions become

$$
\begin{gathered}
\hat{\Phi}_{0} \rightarrow \hat{x}, \\
\hat{\phi}_{0} \rightarrow \frac{\hat{x}}{\hat{r}^{3}}, \\
n_{0} \rightarrow 1 \quad \text { as } \hat{r} \rightarrow \infty .
\end{gathered}
$$

We note that the unit of distance now coincides with the switchover distance, $\hat{\epsilon} \nu^{-1 / 2}$, of Sec. 4.2. When $\tilde{\nu}$ is large, $(4.21 \mathrm{c})$ yields Laplace's equation for $\hat{\phi}_{0}$, to lowest order in $1 / \tilde{\nu}$. Subject to $(4.22 \mathrm{~b})$, and in the absence of quadrupoles or higher, this implies that, to lowest order, we have $\hat{\phi}_{0}=\hat{x} / \hat{r}^{3}$. Hence we have undertaken the numerical experiment of solving

$$
\frac{1}{2}|\nabla \Phi|^{2}+\frac{x}{r^{3}}=\frac{1}{2}
$$

in the whole domain, subject to $\Phi \rightarrow x$ as $r \rightarrow \infty$. The characteristics of this eikonal equation are the trajectories of the ions and they are illustrated in Fig. 5. They are non-intersecting up to an envelope or 'caustic', but the subsequent trajectories are not consistent with the original continuum model.

We can draw an analogy by recalling that when a thin sheet of water flows rapidly from a point source on a plane that can be tilted from the horizontal, then, above a certain slope, the trajectories of the water particles will have an envelope as in Fig. 5, the dipole potential being replaced by the gravitational potential on the plane; the envelope is roughly parabolic downhill [20]. In this situation, the water particles coalesce into a tube rather than staying in a sheet, the tube being thin but with much larger cross-sectional dimensions than the thickness of the sheet. This prompts us to propose the following scenario for large enough values of $\tilde{\nu}$ : it is conceivable that, when $\tilde{\nu}$ is large enough, a strong axi-symmetric jet of ions is formed downstream of the dust particle. In this jet, the ion density per unit cross-sectional area would exceed the corresponding value upstream of the jet by an order of magnitude. Such a jet, if it existed, could be modelled using the theory of delta-shocks, as described in $[21]$, but we will not pursue this possibility further here. 


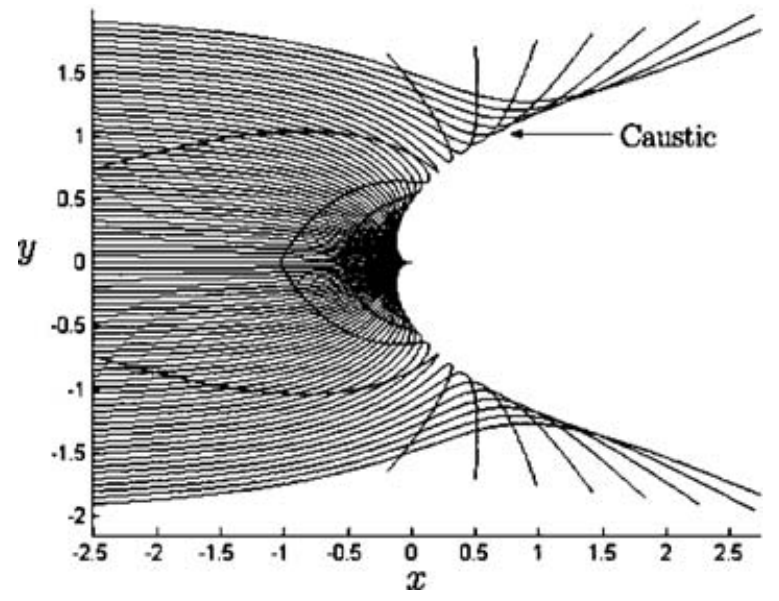

Figure 5. Ion trajectories in a dipole field. The scale length is $\hat{\epsilon} / \nu^{1 / 2}$.

\section{Summary}

We have modelled a spherical grain in a stationary plasma using the ABR theory, which assumes cold, collisionless ions. We exploited the smallness of the grain size compared to the electron Debye length to find matched asymptotic expansions for the electric potential, the ion number density and the ion velocity. One of the main achievements of this asymptotic approach was the discovery of a new shielding length, associated with the ABR model. It depends not only on the electron Debye length but also on the grain size and the ion-electron mass ratio.

We then considered the effect of ion streaming relative to the dust grain, a common scenario in dusty plasmas. For streaming below a certain threshold we determined matched asymptotic expansions for the electric potential, ion number density and ion velocity. To lowest order these are all spherically symmetric inside the shielding distance. At threshold streaming the spherical symmetry inside the shielding distance is lost, and we found the resulting problem to be analytically intractable.

When the streaming exceeds the threshold, it is possible that the ions can no longer re-adjust smoothly to their free stream velocity downstream of the dust particle. This leads us to speculate that they condense into a concentrated jet, but further numerical and experimental evidence is needed before this scenario can be properly discussed.

While sophisticated numerical calculations are undoubtedly useful, it is hoped that the work in this paper demonstrates that techniques such as matched asymptotic expansions can also be valuable, and effective, in gaining new insights into the behaviour of a dust particle in a plasma.

\section{Appendix A. Uniqueness result}

Because (2.14) is a second-order ordinary differential equation, its general solution will have two degrees of freedom. We show first how two degrees of freedom arise from the general solution of (2.14), and then how both of these degrees of freedom are eliminated by the given far-field boundary conditions. 
We begin with a formal, far-field analysis. From (2.15) we can write

$$
\phi \sim-\frac{J^{2}}{2 r^{4}}+\bar{\phi} \quad \text { as } r \rightarrow \infty,
$$

where

$$
\bar{\phi} \sim o\left(r^{-4}\right) \quad \text { as } r \rightarrow \infty .
$$

The linearized equation for $\bar{\phi}$ reads

$$
\frac{d^{2} \bar{\phi}}{d r^{2}}+\frac{2}{r} \frac{d \bar{\phi}}{d r}=-\frac{J^{2}}{2 r^{4}}-\frac{r^{4}}{J^{2}} \bar{\phi} \quad \text { as } r \rightarrow \infty .
$$

This is a second-order ordinary differential equation in $\bar{\phi}$. Any particular integral can be written as an inverse power series beginning with terms of $O\left(r^{-8}\right)$; to find the behaviour of the complementary function as $r \rightarrow \infty$ we write the WKB [21] ansatz

$$
\bar{\phi} \sim A(r) e^{\psi(r)} \quad \text { as } r \rightarrow \infty,
$$

where $|\psi| \rightarrow \infty$ as $r \rightarrow \infty$,

$$
\begin{gathered}
\bar{\phi}^{\prime} \sim A \psi^{\prime} e^{\psi}+A^{\prime} e^{\psi}, \\
\bar{\phi}^{\prime \prime} \sim A \psi^{\prime \prime} e^{\psi}+A\left(\psi^{\prime}\right)^{2} e^{\psi}+2 A^{\prime} \psi^{\prime} e^{\psi},
\end{gathered}
$$

where " denotes $d / d r$. Hence

$$
\left(\psi^{\prime}\right)^{2} \sim-\frac{r^{4}}{J^{2}} \Rightarrow \psi \sim \pm \frac{i r^{3}}{3 J} \quad \text { as } r \rightarrow \infty .
$$

To find $A$ we substitute (A 7) into (A 5) and (A 6). In turn, substituting these into (A 3) and retaining terms in $A^{\prime}$, we find eventually

$$
A^{\prime} r+2 A \sim 0 \Rightarrow A \sim \frac{b}{r^{2}}
$$

for a constant $b$. Combining this with (A 7), we have that the complementary functions are such that

$$
\bar{\phi}_{C} \sim \frac{b_{+}}{r^{2}} \exp \left(\frac{i r^{3}}{3 J}\right)+\frac{b_{-}}{r^{2}} \exp \left(-\frac{i r^{3}}{3 J}\right) \quad \text { as } r \rightarrow \infty,
$$

where $b_{+}$and $b_{-}$are the two arbitrary constants, which both vanish from (A 2).

In summary, using only the information supplied by the far-field condition on $n$, we have shown how both degrees of freedom in the overall problem are eliminated. Thus for a given $J, \phi(r)$ is also uniquely determined.

\section{Acknowledgements}

The first author wishes to acknowledge financial support from EPSRC and QinetiQ. The authors also wish to thank Professor Bryce McLeod for useful discussions.

\section{References}

[1] Selwyn, G. S., Haller, K. L. and Heidenreich, J. E. 1991 Rastered laser light scattering studies during plasma processing: particle contamination trapping phenomena. $J$. Vac. Sci. Technol. A 9, 2824.

[2] Selwyn, G. S., Singh, J. and Bennett, R. S. 1989 In situ laser diagnostic studies of plasma generated particle contamination. J. Vac. Sci. Technol. A 7, 2758. 
[3] Roca i Cabarrocas, J. 2000 Plasma enhanced chemical vapor deposition of amorphous, polymorphous and microcrystalline silicon films. J. Non-Cryst. Solids $\mathbf{2 6 6}, 31$.

[4] Boufendi, L. and Bouchoule, A. 2002 Industrial developments of scientific insights in dusty plasmas. Plasma Sources Sci. Technol. 11, A211.

[5] Goertz, C. K. 1989 Dusty plasmas in the solar system. Rev. Geophys. 27, 271.

[6] Hartquist, T. W., Havnes, O. and Morfill, G. E. 2003 The effects of charged dust on Saturn's rings. Astron. Geophys. 44(5), 5.26.

[7] Fortov, V. E., Ivlev, A. V., Khrapak, S. A., Khrapak, A. G. and Morfill, G. E. 2005 Complex (dusty) plasmas: current status, open issues, perspectives. Phys. Rep. 421, 1.

[8] Konopka, U., Morfill, G. E. and Ratke, L. 2000 Measurement of the interaction potential of microspheres in the sheath of a RF discharge. Phys. Rev. Lett. 84, 891.

[9] Konopka, U., Ratke, L. and Thomas, H. M. 1997 Central collisions of charged dust particles in a plasma. Phys. Rev. Lett. 79, 1269.

[10] Lapenta, G. 2002 Nature of the force field in plasma wakes. Phys. Rev. E 66, 026409-1.

[11] Hutchinson, I. 2005 Ion collection by a sphere in a flowing plasma: 3. Floating potential and drag force. Plasma Phys. Control. Fusion 47, 71.

[12] Kennedy, R. V. and Allen, J. E. 2002 The floating potential of spherical probes and dust grains. Part 1. Radial motion theory. J. Plasma Phys. 57, 243.

[13] Kennedy, R. V. and Allen, J. E. 2003 The floating potential of spherical probes and dust grains II: Orbital motion theory. J. Plasma Phys. 69, 485.

[14] Van Dyke, M. D. 1975 Perturbation Methods in Fluid Mechanics. Stanford, CA: Parabolic Press.

[15] Annaratone, B. M., Allen, M. W. and Allen, J. E. 1992 Ion currents to cylindrical Langmuir probes in RF plasmas. J. Phys. D: Appl. Phys. 25, 417.

[16] Swift, J. D. and Schwar, M. J. R. 1970 Electrical Probes for Plasma Diagnostics. London: Iliffe.

[17] Allen, J. E., Boyd, R. L. F. and Reynolds, P. 1957 The collection of positive ions by a probe immersed in a plasma. Proc. Phys. Soc. London B 70, 297.

[18] Arinaminpathy, N., Allen, J. E. and Ockendon, J. R. 2005 On the effect of isolated dust grains in a plasma. DPhil, Oxford.

[19] Chapman, S. and Cowling, T. G. 1970 The Mathematical Theory of Non-uniform Gases. Cambridge: Cambridge University Press.

[20] Edwards, C., Howison, S., Ockendon, J. R. and Ockendon, H. 2005 Hypercritical shallow water flows. DPhil, Oxford.

[21] Hinch, E. J. 1991 Perturbation Methods (Cambridge Texts in Applied Mathematics). Cambridge: Cambridge University Press. 